\title{
Modelo de pronóstico de precipitación para la ciudad de Managua utilizando redes neuronales artificiales en el contexto de la gestión ambiental
}

\author{
M.Sc. Alina María Ortíz \\ Docente investigadora \\ UNAN-Managua, Facultad de Ciencias e Ingenierías \\ aortiz@unan.edu.ni
}

Fecha de recepción: 31 de octubre, 2019 / Fecha de aceptación: 13 de noviembre, 2019 https://doi.org/10.5377/torreon.v8i22.9028

Palabras clave: modelo de pronóstico, teleconexiones, redes neuronales artificiales, variables climatológicas

\section{RESUMEN}

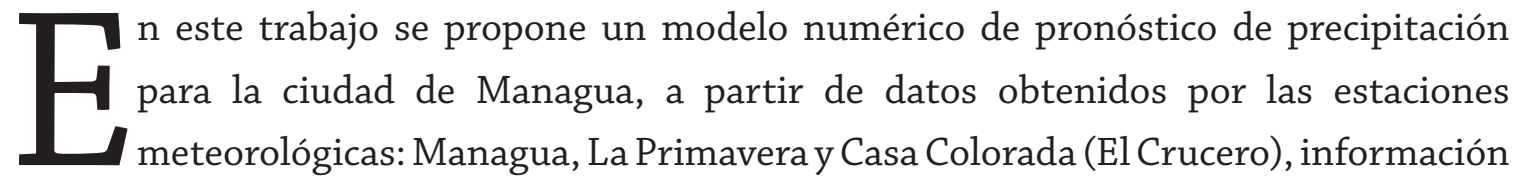
proporcionada por el Instituto Nicaragüense de Estudios Territoriales (INETER), del período correspondiente a 1950-2014 y otras variables climatológicas como la Oscilación Ártica (AO), Oscilación del Atlántico Norte (NAO), Índice de Oscilación del Sur (SOI), Índice de Anomalía de la Media Mensual de la Temperatura de la Superficie del Mar (TSM) en el Atlántico tropical Norte, región $5.5^{\circ} \mathrm{N}-23.5^{\circ} \mathrm{N}$ y $57.5^{\circ} \mathrm{W}-15^{\circ} \mathrm{W}$ (TNA) y el índice oceánico del NIÑO (ONI), a través de teleconexiones. Para la elaboración del modelo se realizó la selección y correlación de variables a través de métodos estadísticos y para encontrar la relación entre estas variables se seleccionó un perceptrón multicapa que es una Red Neuronal Artificial, cuya arquitectura en este caso está compuesta por; una capa de entrada, una capa oculta y una capa de salida. Esta red ha sido entrenada mediante aprendizaje supervisado a través del algoritmo de backpropagation. 
Dicha red se utilizará para predecir condiciones climáticas en la ciudad, lo cual permitirá contribuir con la toma decisiones acerca del manejo y planificación de las actividades sensibles al clima para hacer frente a posibles desastres naturales.

\section{INTRODUCCIÓN}

Algunos de los países de la región de América Central, tales como Honduras y Nicaragua, se encuentran entre los principales países con alto riesgo climático debido a la ocurrencia de eventos climáticos extremos ubicándose en la tercera y cuarta posición dentro del Índice Mundial de Riesgo Climático (IRC) (Harmeling 2010) para el periodo 1990-2009.

En muchos lugares de acuerdo a las condiciones físico geográficas y al cambio climático, la ocurrencia de lluvias viene acompañada de inundaciones catastróficas y de pérdidas humanas, económicas y materiales de toda índole, así como también grandes e incalculables daños a la producción agrícola, (Chourio 2009) Managua tiene una posición geográfica que la ubica en una depresión con 20 kilómetros de zona costera con el lago Xolotlán lo cual la convierte en la zona más vulnerable ante inundaciones en todo el país, (G. Flores 2019). Debido a la orografía montañosa que presenta la ciudad con niveles significativos de deforestación y las lluvias persistentes provocan fácilmente deslizamientos y derrumbes en laderas, contaminación del agua y la consecuente sedimentación y degradación de los suelos agrícolas; y por tanto desastres socio-naturales de gran impacto, (SINAPRED 2010-2015).

Managua es la ciudad más poblada de Nicaragua, con más de un millón de habitantes, en la zona urbana se encuentra la mayor parte de la población la cual representa el 90 por ciento y en la zona rural donde está la menor parte de la población, 10 por ciento, (www.inec.gob.ni/2003).

Cada año durante la temporada de lluvia presenta severas afectaciones por inundaciones en el área urbana, teniendo como principales causas: (Ficha Municipal, INIFOM. 2012),

- El crecimiento urbano acelerado y sin criterios de planificación.

- Cuerpos de agua y zonas contaminadas.

- Falta de responsabilidad y cultura ambiental y urbanística en la población.

- Viejo y deficiente sistema de drenaje pluvial.

- Utilización de los cauces como basureros.

Sumado a todo esto se puede agregar que Managua recibe el agua que desciende de las partes altas de la cuenca que drenan hacia el interior a través del sistema de cauces de desagüe que atraviesan la Ciudad hacia su desembocadura en el lago. 
En Nicaragua, se utilizan varios modelos de predicción climática a escala global, (INETER 2016), es por ello que se propone un modelo predictivo que permita el monitoreo local de la precipitación lo cual genere pronósticos acertados, permitiendo una respuesta ágil que minimice el nivel de amenaza para el departamento cuya vulnerabilidad es grande.

\section{Objetivo}

Correlacionar variables climatológicas para la generación de un modelo de pronóstico de precipitación utilizando las RNA, para el departamento de Managua en el contexto de la gestión ambiental.

\section{MATERIAL Y MÉTODO}

En la realización de este estudio se utilizaron tres estaciones meteorológicas:

Estación Meteorológica Principal Managua (HMP): Está ubicada en el Departamento de Managua, en el municipio de Managua (costado este Aeropuerto Internacional Managua). Se encuentra en el cuadrante de Nindirí C2952 II. Código 069027, Latitud Norte 1208’36”, Longitud Oeste 8609’49”, elevación 56 msnm.

Estación meteorológica La Primavera (PV): Está ubicada en el departamento de Managua, en el municipio de Managua. Código 069046, Latitud Norte 1201'08', Longitud Oeste 86¹4'55”, elevación 600 msnm.

Estación meteorológica Casa colorada PV (E1 Crucero): Está ubicada en el municipio de El Crucero. Código 069047, Latitud Norte 1158'54”, Longitud Oeste 86¹8’36”, elevación 910 msnm.

Todas representativas del área de estudio con la información mensual de los diferentes parámetros para un período de 64 años (1950-2014).

Se definió la precipitación como la variable climática local del tiempo a utilizar y a escala global, la temperatura, la presión atmosférica, la humedad, la nubosidad, el viento, contempladas en los índices oceánicos a través de la correlación, positiva o negativa entre varios índices atmosféricos que proporcionan datos sobre: la Oscilación Ártica (AO), Oscilación del Atlántico Norte (NAO), Índice de Oscilación del Sur (SOI), Índice de Anomalía de la media mensual de la Temperatura de la superficie del mar (TSM) en el Atlántico tropical Norte, región $5.5^{\circ} \mathrm{N}-23.5^{\circ} \mathrm{N}$ y $57.5^{\circ} \mathrm{W}-15^{\circ} \mathrm{W}$ (TNA) y el Índice Oceánico del NIÑO (ONI), Oscilación del Sur (ENSO), lo que permite enlazar los cambios del tiempo que ocurren en regiones separadas por grandes distancias del globo terráqueo con la lluvia en la zona de estudio usando como marco de correlación los datos de precipitación aplicado a la variabilidad en escalas de tiempo. 
Luego se procedió a verificar si los datos de índice atmosférico y precipitación son ajustables a través del análisis de funciones de probabilidades hasta obtener la que mejor se ajuste.

Se realizó la correlación de las variables como una serie de tiempo cuya tendencia dada por el movimiento general a largo plazo de la información presenta una tendencia no lineal, seleccionando de las tendencias no lineales la exponencial, ya que es la que mejor se ajusta a la distribución de puntos y la que mayores coeficientes de correlación presentan. Una vez determinada la correlación entre las variables utilizando los datos registrados de las estaciones meteorológicas mencionadas se utilizó alimentación hacia adelante con redes neuronales artificiales para el aprendizaje supervisado.

El funcionamiento de la red consiste en un aprendizaje de un conjunto predefinido de pares de entradas-salidas empleando un ciclo propagación-adaptación de dos fases:

1. Se aplica un patrón de entrada como estímulo para la primera capa de las neuronas de la red luego se va propagando a través de todas las capas superiores hasta generar una salida y se compara el resultado obtenido en las neuronas de salida con la salida que se desea obtener y se calcula un valor del error para cada neurona de salida.

2. Estos errores se transmiten hacia atrás, partiendo de la capa de salida, hacia todas las neuronas de la capa intermedia que contribuyan directamente a la salida de esa forma recibiendo el porcentaje de error aproximado a la participación de la neurona intermedia en la salida original. Este proceso se repite, capa por capa, hasta que todas las neuronas de la red hayan recibido un error que describa su aportación relativa al error total tomando como base el valor del error recibido, se reajustan los pesos de conexión de cada neurona de manera que en la siguiente vez que se presente el mismo patrón la salida esté más cercana a la deseada (disminuya el error). Los pasos se repiten hasta alcanzar un mínimo del error de entrenamiento para lo cual se realizan m ciclos de aprendizaje.

Ya entrenada la red se podrá aplicar esa misma relación (finalizado el entrenamiento) a nuevos vectores de entrada dando una salida activa si la nueva entrada es parecida a las presentadas durante el aprendizaje. La red neuronal a entrenar será utilizada para predecir condiciones climáticas en Managua.

Finalmente se proponen medidas de mitigación que permitan un manejo integrado de la Ciudad. 


\section{RESULTADOS Y DISCUSIÓN}

A continuación, se presenta la correlación de cada uno de los índices atmosféricos AO, TNA, NAO, ONI Y SOI con el promedio mensual de precipitación en cada una de las estaciones a lo largo de los 64 años de que se disponen datos.

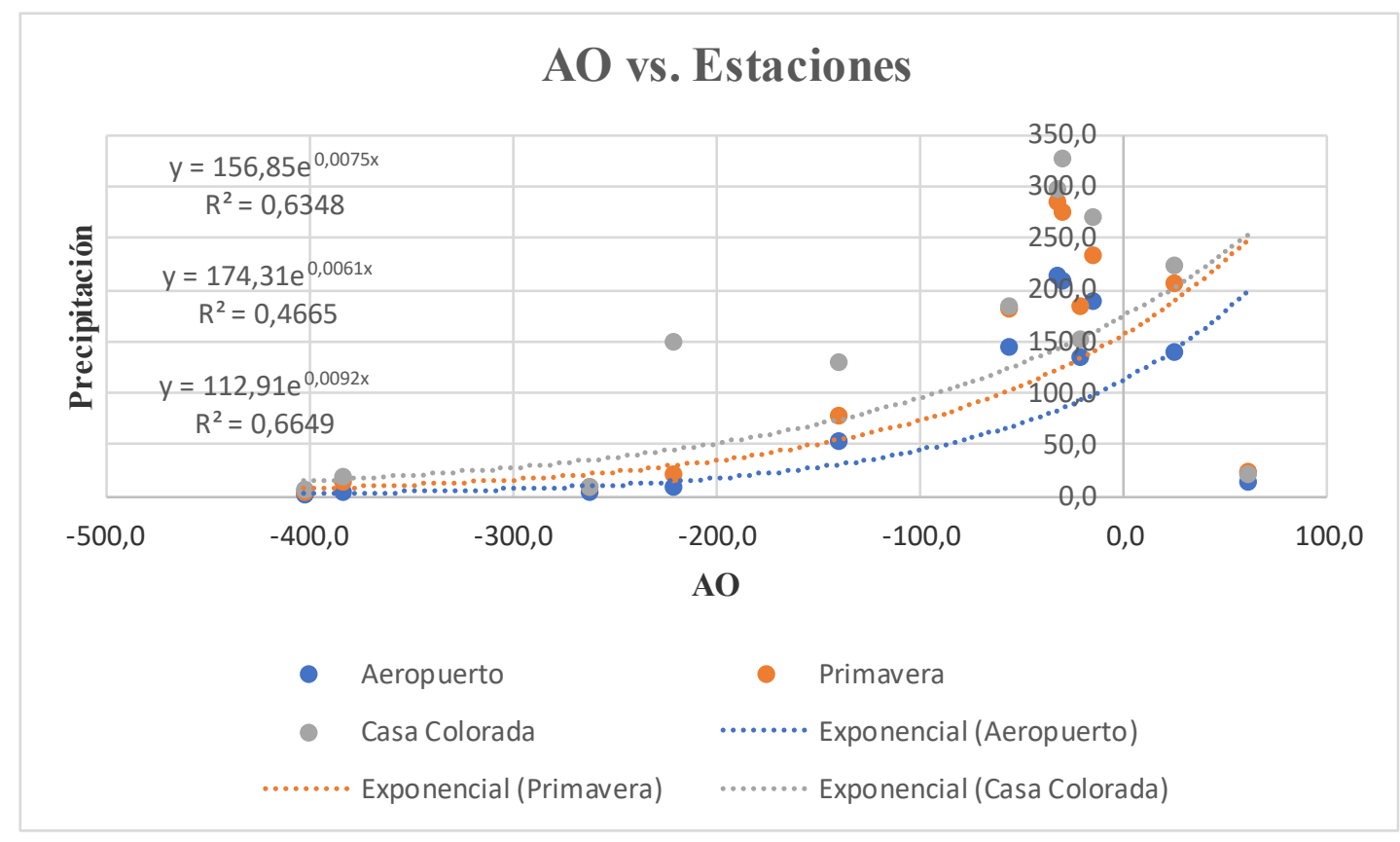

Figura 1. Oscilación AO versus estaciones Aeropuerto, Primavera y Casa colorada 


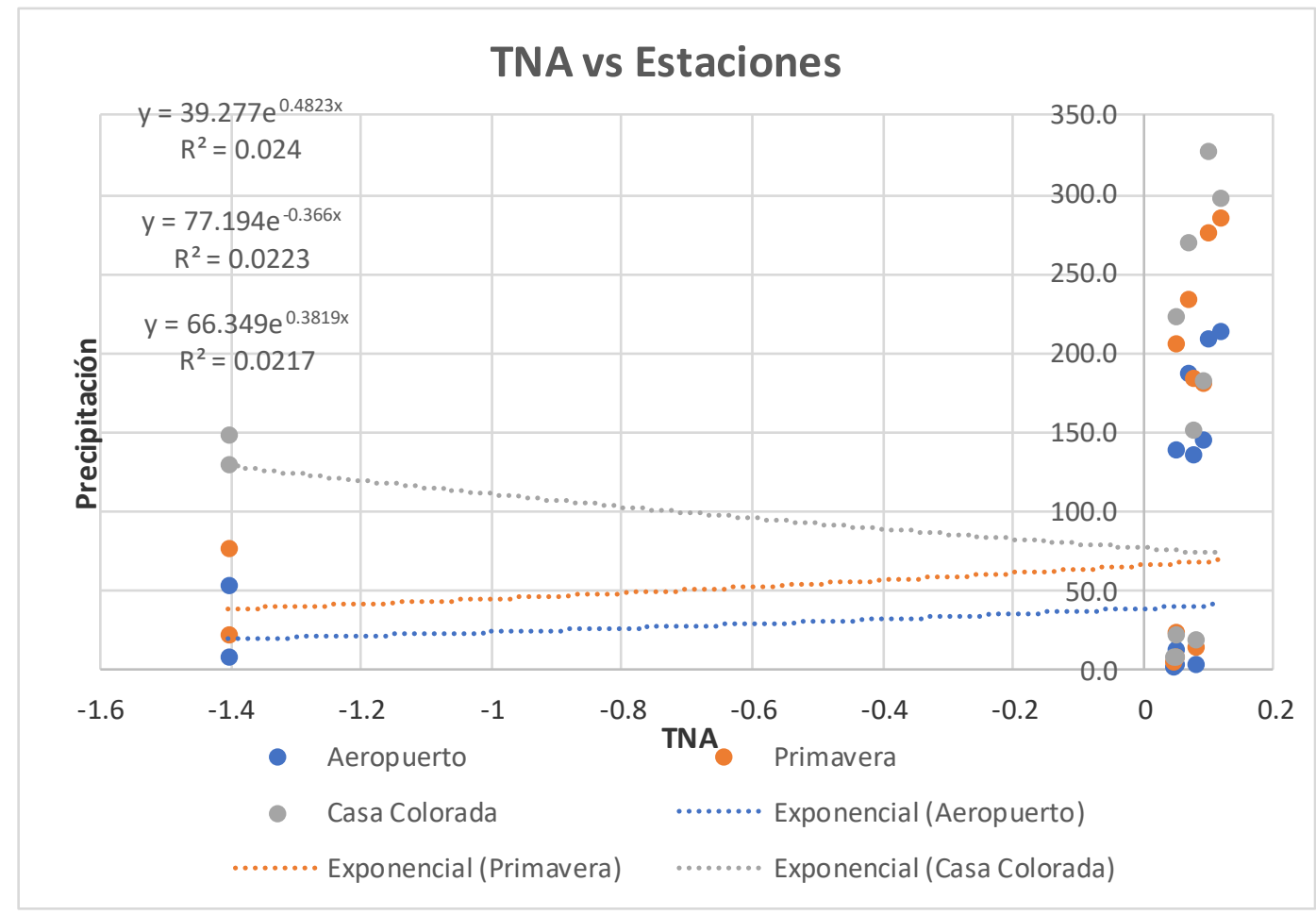

Figura 2. Gráfico Oscilación TNA versus estaciones Aeropuerto, Primavera y Casa colorada

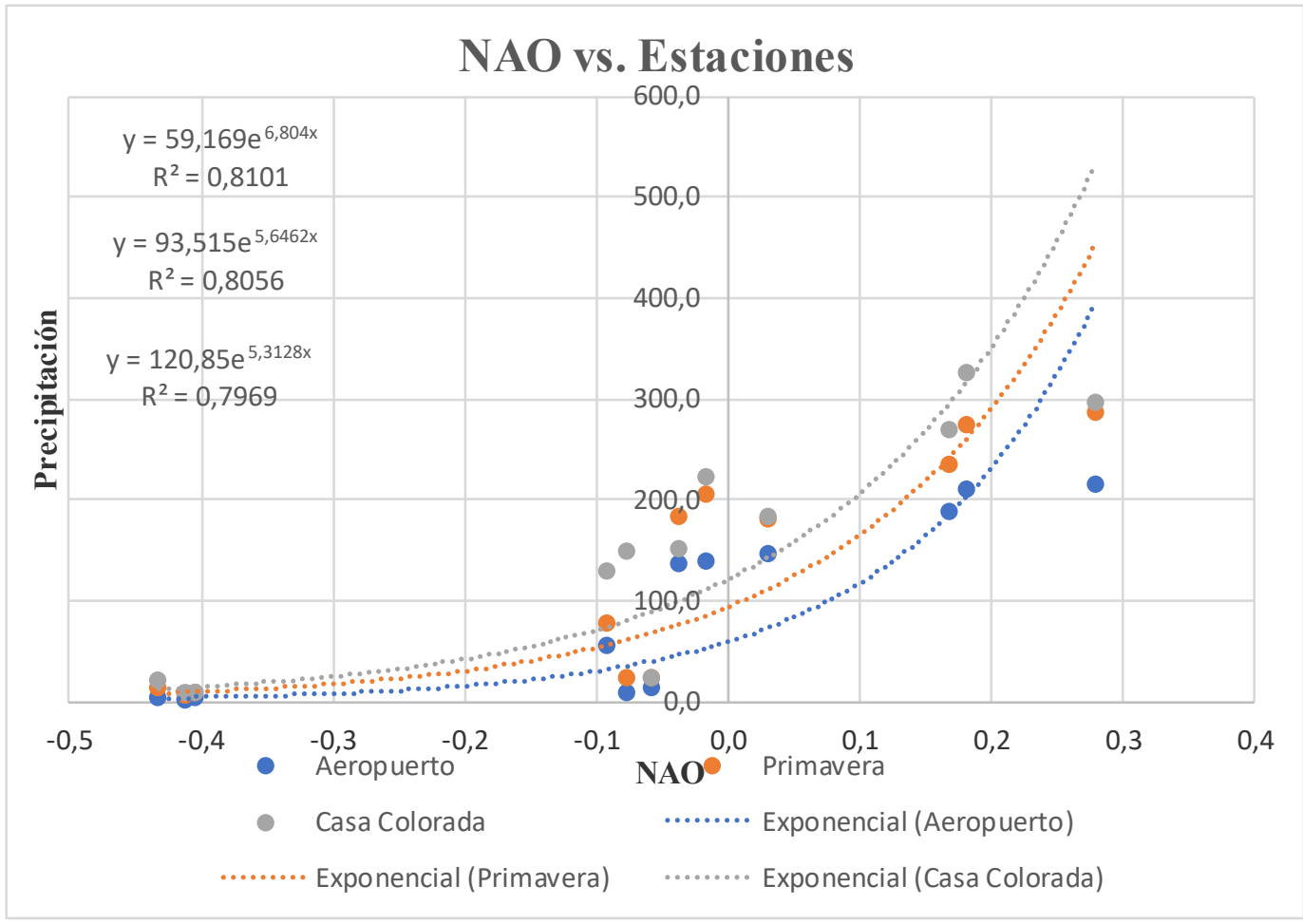

Figura 3. Gráfico Oscilación NAO versus estaciones Aeropuerto, Primavera y Casa colorada 


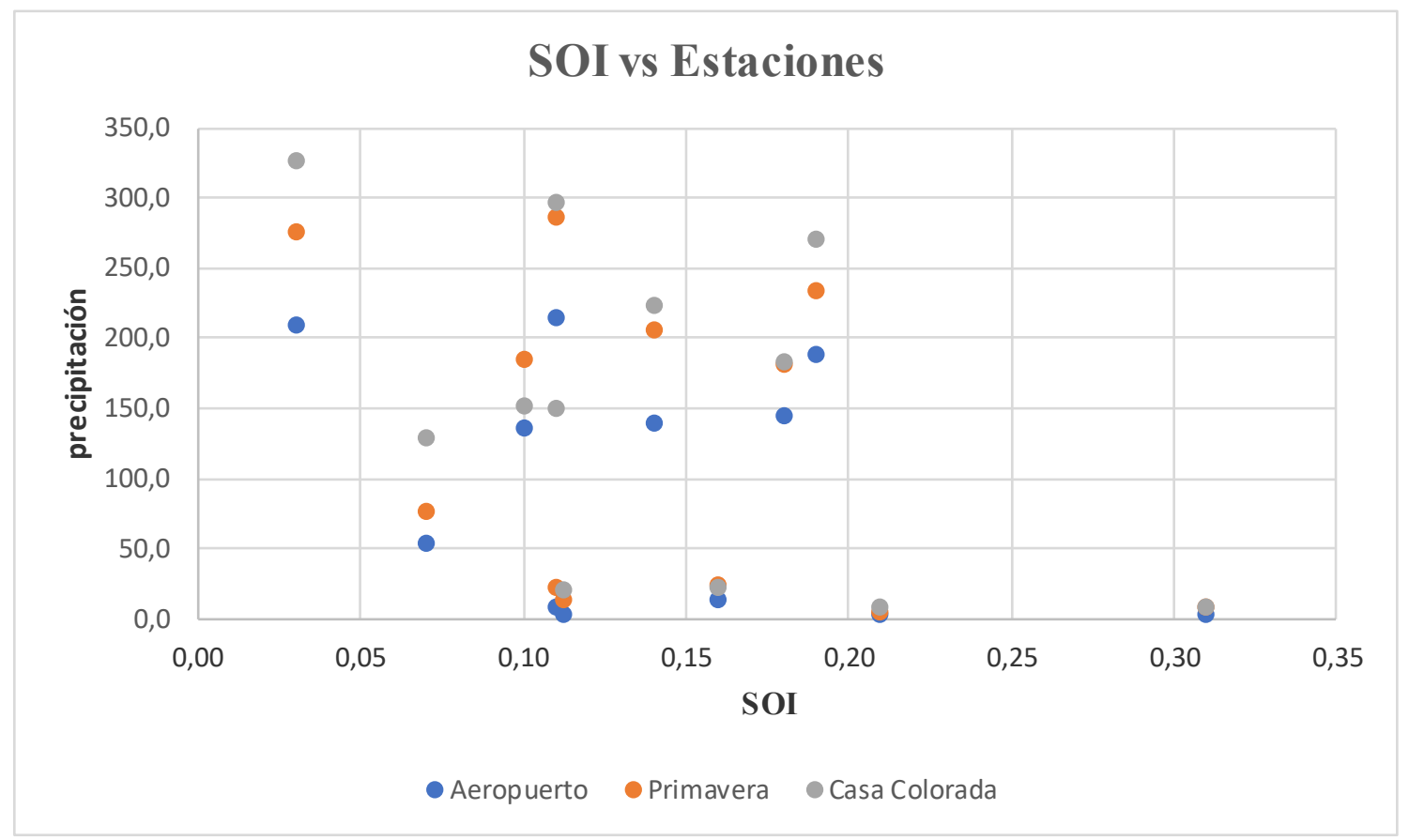

Figura 4. Gráfico Oscilación SOI versus estaciones Aeropuerto, Primavera y Casa colorada

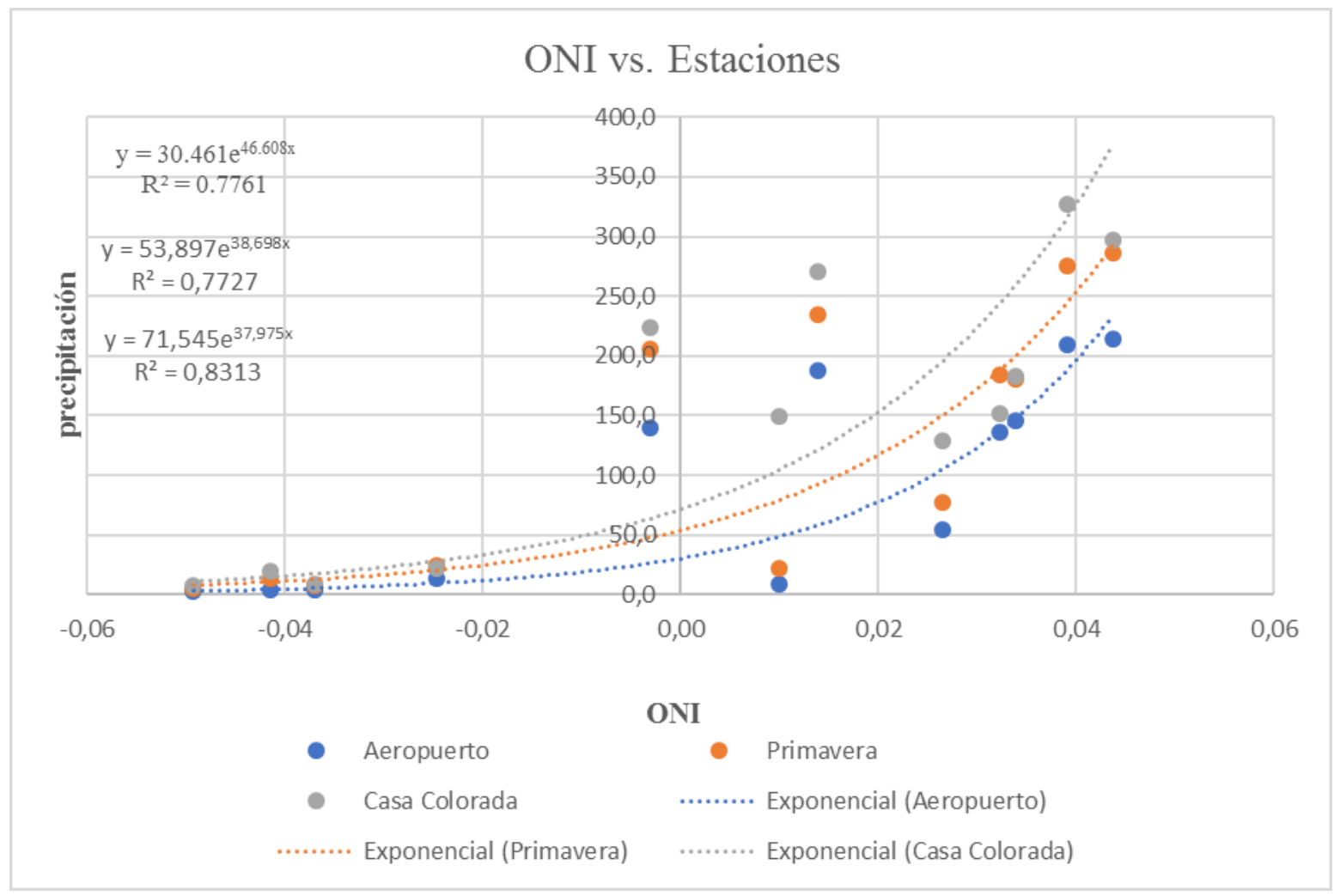

Figura 5. Gráfico Oscilación ONI versus estaciones Aeropuerto, Primavera y Casa colorada

En las figuras mostradas es posible observar que la serie de tiempo presenta una tendencia no lineal, este comportamiento curvilíneo, sin embargo, muestra que mientras más pequeño es 
el valor del índice atmosférico menor es la precipitación y que a medida que aumenta el valor de este también aumenta la precipitación. En la figura 4 la distribución de puntos es aleatoria por lo cual no es posible ajustar dicha distribución.

Se realizó la correlación de las variables como una serie de tiempo ya que se tienen datos registrados en forma periódica cuya tendencia es no lineal. Entre las tendencias no lineales que pueden presentarse en una serie de datos se encuentran, la polinomial, logarítmica, exponencial y potencial, entre otras, de estas la que mejor se ajusta a la distribución de puntos es la tendencia exponencial, y la que mayores coeficientes de correlación presentan, lo que permitió encontrar los Mejores Estimadores Insesgados.

El modelo de pronóstico propuesto basado en características específicas para el comportamiento de la precipitación en el Departamento de Managua considera la selección de las variables climatológicas a utilizar; en este caso los datos de precipitación de tres estaciones climatológicas y los índices climatológicos a lo largo de un periodo de 64 años, la correlación de las variables involucradas a través de series temporales y la normalización de los valores máximos, medios y mínimos de la precipitación y de los índices climatológicos, los cuales se convierten en los valores de salida y entrada respectivamente para la red neuronal definida.

Se propone una red neuronal de tipo perceptrón multicapas que optimiza tanto la matriz de pesos como el número de neuronas ocultas, cuya arquitectura está compuesta por una capa de entrada, una capa oculta y una capa de salida. En el modelo se parte de una red neuronal $(2,2,1)$ que se identifica con dos neuronas en la capa de entrada, dos en la capa oculta y una en la capa de salida con el objetivo de optimizar la matriz de pesos mediante el algoritmo backpropagation y tres variables que identifican al modelo. Los pesos, son los coeficientes que almacenan el conocimiento necesario para resolver problemas específicos, en este caso las predicciones.

La ciudad de Managua y el país en general pueden gestionar los riesgos de desastre con mayor eficacia si se incluyen aspectos relativos a esos riesgos en los planes de desarrollo tanto a nivel sectorial como a nivel nacional y si se adoptan estrategias de adaptación al cambio climático traduciendo esos planes y estrategias en medidas destinadas a zonas y grupos vulnerables. Entre las que se pueden mencionar:

- La evaluación de riesgos, ubicación de la amenaza potencial y su grado de severidad.

- Sistemas de alerta temprana.

- La comunicación entre las instancias decisorias y los ciudadanos locales en relación a los riesgos.

- Integrar la gestión de los recursos hídricos y la planificación y diseño de sistemas en políticas sobre el uso de suelos. 
- La gestión y restauración de los ecosistemas.

- Mejoras en la vigilancia de la salud pública, el suministro de agua y los sistemas de saneamiento, irrigación y drenaje.

- Desarrollo y aplicación de normas para la construcción.

- Mayor educación y sensibilización en relación a estos temas.

- Gestión de cuencas, fomentando por ejemplo, las buenas prácticas agroforestales sostenibles, la conservación del suelo y las medidas de control de incendios.

- Crear un marco institucional adecuado, así como los mecanismos para poner en práctica las medidas de reducción de la vulnerabilidad.

- Adaptar la agricultura para protegerse ante los cambios climáticos locales.

- Afianzar la seguridad alimentaria.

- Equilibrar las demandas de energía.

- Fortalecer el engranaje entre todas las estructuras del Sistema Nacional para la Prevención, Mitigación y Atención de Desastres y en todos los niveles del territorio nacional.

Es necesario mencionar que esta es una primera aproximación a la propuesta del modelo de predicción; deberán realizarse otras pruebas con el objetivo de verificar la certeza y precisión de las predicciones que se obtienen o hacer modificaciones de ser necesario.

\section{AGRADECIMIENTOS}

La autora agradece a la Dirección de Posgrado de la Universidad Nacional Autónoma de Nicaragua (UNAN-Managua), a través del Fondo de Proyectos de Investigación (FPI) por el financiamiento de la presente investigación.

\section{REFERENCIAS}

CEPAL, (Comisión Económica para América Latina y el Caribe). 2010. Nicaragua efectos costa de América Latina y el Caribe. Dinámicas, tendencias y variabilidad climática., diciembre de 2011. Retomado de https://www.cepal. org/es/publicaciones/3953-efectos-cambioclimatico-la-costa-america-latina-caribe-guiametodologica

Chávez Espíritu, Tulio W. 2012. Análisis hidrológico ante impactos del cambio climático y cambios de uso del suelo en la 
cuenca del río Compasagua, Nicaragua. Tesis MSc. Turrialba, CR, CATIE. Retomado de http://repositorio.bibliotecaorton.catie.ac.cr/ handle/11554/4471

Obregón, N.*, Fragala, F.* y Prada, L. F.** Seminario Internacional La Hidroinformática en la gestión integrada de los recursos hídricos. REDES NEURONALES ARTIFICIALES EN HIDROINFORMÁTICA. Pontificia Universidad Javeriana. Bogotá, Colombia. Universidad de los Andes. Bogotá, Colombia. Retomado de http://cinara.univalle.edu.co/ archivos/pdf/61.pdf

Instituto Nicaragüense de Estudios Territoriales (INETER). Dirección general de Meteorología. Caracterización climática del Departamento de Managua. Dirección de aplicaciones de la meteorología Managua, 11 de diciembre 2008. Año: 2004 Editorial: Monografías del Instituto Nacional de Meteorología Páginas: 279 Ciudad: Madrid. Retomado de https://servmet.ineter. gob.ni//Meteorologia/climadenicaragua.php

Hilera, J.R., y V.J. Martínez. 2000. Redes neuronales artificiales. Fundamentos, modelos y aplicaciones. 390 p. Editorial Alfaomega RaMa, Madrid, España. https://www.academia. edu/39737539/REDES_NEURONALES_ ARTIFICIALES_FUNDAMENTOS _ MODELOS_Y_APLICACIONES

Ovando, Gustavo, Bocco, Mónica, \& Sayago, Silvina. 2005. REDES NEURONALES PARA MODELAR PREDICCIÓN DE HELADAS. Agricultura Técnica, 65(1), 65-73.

Retomado de http://www.scielo.cl/scielo. php?script $=$ sci_arttext \&pid=S0365-
$28072005000100007 \& \operatorname{lng}=$ es \&tlng=es . 10.4067/S0365-28072005000100007.

Ribalaygua, J., De Loma-Ossorio, E., Córdoba, M., Torres, L., Lahoz, C., Arias, A. y Caicedo, A. MOPT. 1992. Guías para la elaboración de estudios del medio físico (contenido y metodología). Ministerio de obras públicas, transporte y medio ambiente. España (2011).

Milán, J. 2011. Apuntes sobre el cambio climático en Nicaragua. Managua, Nicaragua. Retomado de http://www.cambioclimatico.ineter.gob.ni/ bibliografia/Educacion\%20y\%20Cambio\%20 Clim\%C3\%A1tico/Apuntes\%20sobre\%20 Cambio\%20Climatico\%20en\%20Nicaragua1. pdf

IPCC (Intergovernmental Panel on Climate Change). 2007. Cambio climático 2007: informe de síntesis. Contribución de los grupos de trabajo I, II y III al cuarto informe de evaluación del Grupo Intergubernamental de Expertos sobre el Cambio Climático, Ginebra, pág. 104. Retomado de https://www.ipcc.ch/ site/assets/uploads/2018/02/ar4_syr_sp.pdf

INETER (Instituto Nacional de Estudios Territoriales). 2005. Normas históricas. "Comportamiento de las temperaturas medias anual en periodo 1971 al 2000". Managua, Nicaragua. Retomado de https:// servmet.ineter.gob.ni//Meteorologia/ climadenicaragua.php

Freeman, J.A., and D.M. Sapera. 1991. Neural networks: algorithms, applications and programming techniques (Computation and Neural Systems Series) 401 p. Addison-Wesley Pub. Co., Reading, Massachusetts, USA. 